\title{
Development of High Resolution Resonance Ionization Mass Spectrometry for Neutron Dosimetry Technique with ${ }^{93} \mathrm{Nb}\left(\mathrm{n}, \mathrm{n}^{\prime}\right){ }^{93 \mathrm{~m}} \mathrm{Nb}$ Reaction
}

\author{
Hideki Tomita ${ }^{1,2, a}$, Takaaki Takatsuka ${ }^{1}$, Takahide Takamatsu ${ }^{1}$, Yoshitaka Adachi ${ }^{1,2}$, Yujin Furuta ${ }^{1}$, \\ Takuma Noto $^{1,2}$, Tetsuo Iguchi ${ }^{1}$, Volker Sonnenschein ${ }^{3}$, Klaus Wendt ${ }^{4}$, Chikara Ito $^{5}$, and Shigetaka \\ Maeda $^{5}$ \\ ${ }^{1}$ Department of Quantum Engineering, Nagoya University, 464-8603 Nagoya, Japan \\ ${ }^{2}$ RIKEN Nishina Center, 2-1 Hirosawa, Wako, Saitama 351-0198, Japan \\ ${ }^{3}$ Department of Physics, University of Jyväskylä, SF-40351, Finland \\ ${ }^{4}$ Institute of Physics, Johannes Gutenberg-University Mainz, Staudingerweg 7, 55128 Mainz, \\ Germany \\ ${ }^{5}$ Oarai Research and Development Center, Japan Atomic Energy Agency, 4002 Narita, Oarai, Ibaraki, \\ 311-1393, Japan
}

\begin{abstract}
We have proposed an advanced technique to measure the ${ }^{93 \mathrm{~m}} \mathrm{Nb}$ yield precisely by Resonance Ionization Mass Spectrometry, instead of conventional characteristic $\mathrm{X}$-ray spectroscopy. ${ }^{93 \mathrm{~m}} \mathrm{Nb}$-selective resonance ionization is achievable by distinguishing the hyperfine splitting of the atomic energy levels between ${ }^{93} \mathrm{Nb}$ and ${ }^{93 \mathrm{~m}} \mathrm{Nb}$ at high resolution. In advance of ${ }^{93 \mathrm{~m}} \mathrm{Nb}$ detection, we could successfully demonstrate high resolution resonant ionization spectroscopy of stable ${ }^{93} \mathrm{Nb}$ using an all solid-state, narrowband and tunable Ti:Sapphire laser system operated at $1 \mathrm{kHz}$ repetition rate.
\end{abstract}

\section{Introduction}

Neutron dosimetry in a nuclear reactor is very important to evaluate the irradiation damage of the reactor vessel, structural component and equipment. Conventionally, the neutron activation method has been applied to evaluate neutron dose, which is based on gamma-ray spectrometry of nuclear reaction products. However, nuclear reaction products with short half-lives of less than ten years are not suitable for monitoring the integrated neutron dose over the reactor life time around 40 years or more by the neutron activation method. Alternatively, retrospective nuclear reactor dosimetry has been proposed, which is based on trace analysis of long-lived and stable isotopes produced through nuclear reactions. ${ }^{93} \mathrm{Nb}\left(\mathrm{n}, \mathrm{n}^{\prime}\right)^{93 \mathrm{~m}} \mathrm{Nb}$ reaction is one of the promising candidates for the dosimetry because it is a threshold reaction desired for measurement of fast neutron fluence and its reaction product ${ }^{93 \mathrm{~m}} \mathrm{Nb}$ has a suitably long half-life of 16.13 years [1].

\footnotetext{
${ }^{a}$ Corresponding author: tomita@nagoya-u.jp
}

This is an Open Access article distributed under the terms of the Creative Commons Attribution License 2.0, which permits unrestricted use, distribution, and reproduction in any medium, provided the original work is properly cited. 
Resonance ionization mass spectrometry (RIMS), which is based on efficient excitation and ionization via atomic energy levels by tunable laser and subsequent mass spectrometry, is widely applied for trace determination of long-lived radioactive isotopes [2-4]. RIMS for measurement of ${ }^{93 \mathrm{~m}} \mathrm{Nb}$ yield was proposed so far [5-7] instead of conventional characteristic X-ray spectroscopy. ${ }^{93 \mathrm{~m}} \mathrm{Nb}$-selective resonance ionization is achievable by distinguishing the hyperfine splitting of the atomic energy levels between ${ }^{93} \mathrm{Nb}$ and ${ }^{93 \mathrm{~m}} \mathrm{Nb}$ at high resolution, which depends on ${ }^{93 \mathrm{~m}} \mathrm{Nb}$ nuclear spin of $1 / 2^{-}$and ${ }^{93} \mathrm{Nb}$ nuclear spin of $9 / 2^{+}$respectively. In this method, cooling time to reduce interference from impurity radioisotopes and complex sample preparation before ${ }^{93 \mathrm{~m}} \mathrm{Nb}$ measurement would be not necessary. Hyperfine splitting of stable ${ }^{93} \mathrm{Nb}$ was investigated and the expected selectivity on ${ }^{93} \mathrm{Nb} /{ }^{93 \mathrm{~m}} \mathrm{Nb}$ measurement was already estimated [5-7]. The investigation was based on resonance ionization spectroscopy using conventional dye laser system. To obtain high ion count rate via ${ }^{93 \mathrm{~m}} \mathrm{Nb}$-selective resonance ionization, a narrow band-width tunable laser operated at high repetition rate should be used for resonant ionization. However, conventional dye laser system operated at high repetition rate is not suitable for stable long-term operation because degradation of laser dye during operation should be considered carefully.

We developed an all solid-state, narrow-band and tunable Ti:Sapphire laser system, which is based on injection locking [8,9], operated at repetition rate up to $10 \mathrm{kHz}$. In addition, some schemes for ${ }^{93 \mathrm{~m}} \mathrm{Nb}$ selective resonance ionization using this laser system was already found [10]. In advance of ${ }^{93} \mathrm{mb}$ detection, we have investigated high resolution resonance ionization spectroscopy of stable ${ }^{93} \mathrm{Nb}$ using the narrow-band and high repetition rate Ti:Sapphire laser system.

\section{Experimental Setup}

Figure 1 shows experimental setup for high resolution resonance ionization spectroscopy of stable ${ }^{93} \mathrm{Nb}$ using Ti:Sapphire laser system. Two color - one resonance excitation step scheme, i.e. resonance excitation and following non-resonance ionization scheme was used as shown in Figure 2. Second harmonic generation (SHG) of narrow-band Ti:Sapphire laser called injection locked Ti:Sapphire laser and standard Ti:Sapphire laser [11] were used for 1st step resonance excitation (wavelength of $374.345 \mathrm{~nm}$ ) and non-resonance ionization (wavelength of $357.6 \mathrm{~nm}$ ) respectively. In this experiment, extended cavity diode laser (ECDL) was used as a master laser of the injection locked Ti:Sapphire laser. The detailed description of the injection locked Ti:Sapphire laser was given in previous papers [5, 6]. The standard Ti:Sapphire laser and the narrow-band Ti:Sapphire laser were pumped by SHG of Nd:YAG laser at repetition rate of $1 \mathrm{kHz}$. Typical band widths of the standard Ti:Sapphire laser and the narrowband Ti:Sapphire laser were $3-5 \mathrm{GHz}$ and $20 \mathrm{MHz}$ respectively. Inside a vacuum chamber, collimated $\mathrm{Nb}$ atom vapor was produced by a hot $\mathrm{Nb}$ filament by ohmic heating with a collimator in the diameter of $6.0 \mathrm{~mm}$ and introduced into ionization region between two electrodes. $\mathrm{Nb}$ atoms were ionized by irradiation of two pulses from the injection locked Ti:Sapphire laser and the standard Ti:Sapphire laser. $\mathrm{Nb}$ ions were accelerated by electrostatic fields between electrodes, and then detected by a multi-channel plate (MCP) after $0.6 \mathrm{~m}$ field free flight from the ionization region. Ions were counted with time gate of flight time of $\mathrm{Nb}$ ion. During ions counting, emission frequency of ECDL, i.e. the frequency of the injection locked Ti:Sapphire laser, was measured by a wavelength meter.

\section{Results and Discussion}

The spectrum of the detected ions was obtained by resonant ionization using the injection locked Ti:Sapphire laser. As a reference, broad spectrum was also obtained using additional standard Ti:Sapphire laser for 1st step instead of the injection locked Ti:Sapphire laser. Figure 3 shows the resonance ionization spectra obtained by frequency scanning of the injection locked Ti:Sapphire laser and the standard Ti:Sapphire laser, respectively. The spectra involved six peaks due to hyperfine 


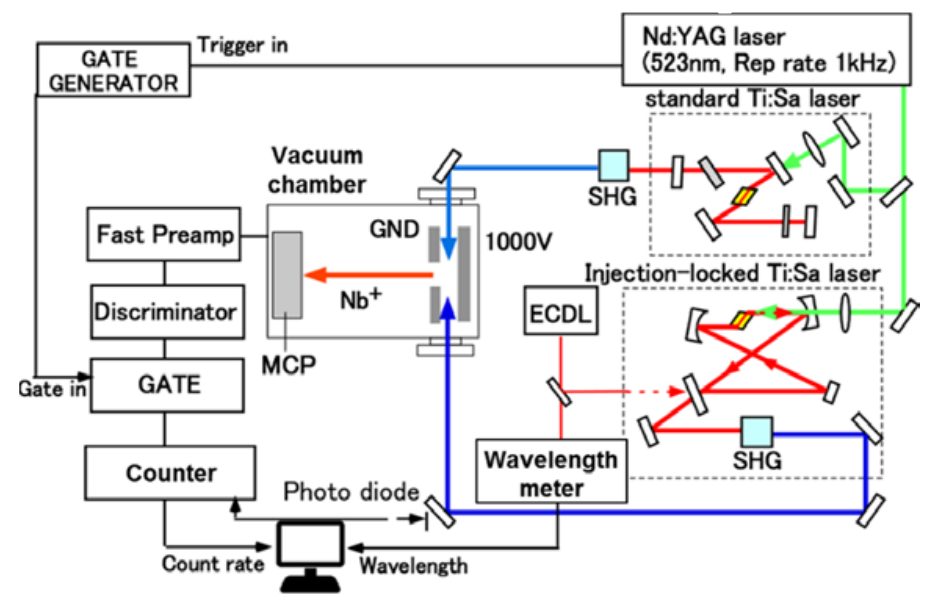

Figure 1. Experimental setup for high resolution resonance ionization spectroscopy of stable ${ }^{93} \mathrm{Nb}$.

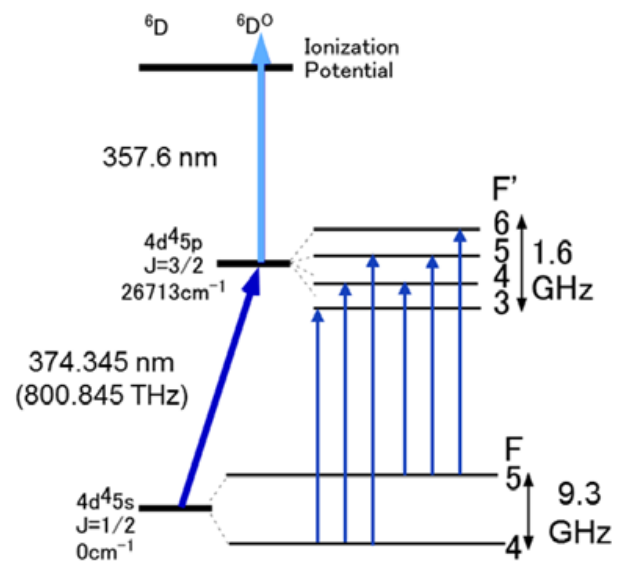

Figure 2. Resonance excitation and ionization scheme of ${ }^{93} \mathrm{Nb}$.

structures in the lower and upper levels as indicated schematically in Fig. 2. Although the broad spectrum without any hyperfine components was obtained using the standard Ti:Sapphire laser, six peaks were clearly resolved in the spectrum obtained by the injection locked Ti:Sapphire laser. The high resolution spectrum was fit with six Voigt functions as shown in Fig. 3(b). Although Lorentzian component of Voigt fitting due to power broadening in 1st step was not negligible, laser power for 1st step can be reduced in the case of more efficient scheme via additional resonance excitation. Thus, Doppler broadening in atomic vapor was dominant in the line width of the spectrum. In the present setup, average line width of Gaussian component of Voigt fitting was estimated to be $220 \mathrm{MHz}$. Assuming that additional step via 2nd excited level such as $4 \mathrm{~d}^{3} 5 \mathrm{~s} 6 \mathrm{~s}^{6} \mathrm{~F}_{1 / 2}$ at $37871.3 \mathrm{~cm}^{-1}$ by the injection locked Ti:Sapphire laser was used, selectivity in ${ }^{93 \mathrm{~m}} \mathrm{Nb}$ resonance ionization of $10^{5}$ would be achievable. Uncertainty of a few percent would be possible if some correction for laser frequency is applied [12]. In addition, systematic error would be reduced because the ratio of ${ }^{93 \mathrm{~m}} \mathrm{Nb} /{ }^{93} \mathrm{Nb}$ is directly measured by RIMS. 

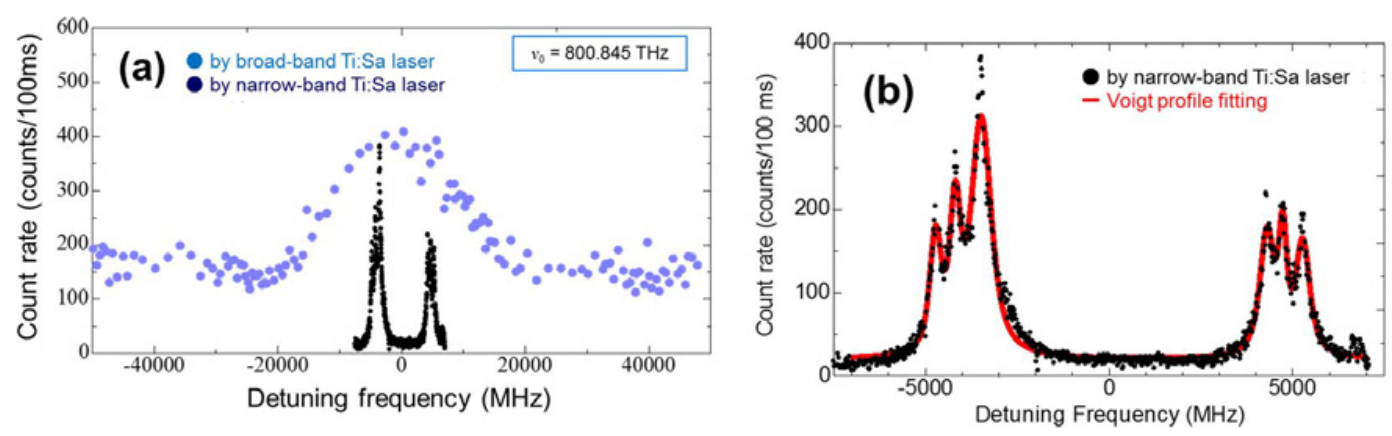

Figure 3. (a) Resonance ionization spectra obtained by frequency scanning of the injection locked Ti:Sapphire laser and the standard Ti:Sapphire laser respectively, (b) high resolution spectrum with six Voigt profile fitting. The center frequency of the injection locked Ti:Sapphire laser was $800.845 \mathrm{THz}(374.345 \mathrm{~nm})$.

\section{Conclusions and Future Work}

High resolution resonance ionization spectroscopy method by high repetition rate Ti:Sapphire laser system was developed toward precise ${ }^{93 \mathrm{~m}} \mathrm{Nb}$ yield measurement. We successfully demonstrated high resolution resonance ionization spectroscopy of stable $\mathrm{Nb}$ using the present laser system, in which hyperfine splitting was resolved clearly. Such high resolution spectroscopy is required for optical separation of ${ }^{93 \mathrm{~m}} \mathrm{Nb}$ signal from stable ${ }^{93} \mathrm{Nb}$ signal and expected selectivity would be $10^{5}$. Uncertainty in isotope ratio measurement by RIMS would be expected less than a few percent with proper correction method applied. Isotope analysis by high resolution RIMS might possibly be applied to the transmutation detectors used for neutron fluence measurement $[13,14]$.

High resolution spectroscopy using additional narrow-band Ti:Sapphire laser will be performed to demonstrate the optical selectivity of $10^{5}$. We are now developing a supersonic gas-jet atomic source to make uniform velocity of sample atoms for higher efficiency resulting from better spatial overlap with laser irradiation and also for higher selectivity resulting suppression of Doppler broadening in the spectroscopy [15]. Gas-jet atomic source will also be involved. In addition, overall efficiency of the measurement will be verified experimentally with $\mathrm{Nb}$ dosimeters of the fast experimental reactor JOYO at Japan Atomic Energy Agency, Japan.

This work was supported by JSPS KAKENHI Grant Number 26420868.

\section{References}

[1] L. Greenwood, et al., J. Nucl. Mater, 361, 1-9 (2007)

[2] V. S. Lethokov, Laser Photoionization Spectroscopy, Academic Press, Orlando (1987)

[3] G. S. Hurst and M. G. Payne, Principles and Application of Resonance Ionisation Spectroscopy, Adam Hilger, Bristol (1988)

[4] Radioisotope Mass Spectrometry in Handbook of Radioactivity Analysis, Edit. M. Annunziata, Academic Press, Amsterdam, 799-845 (2003)

[5] H.M. Lauranto, et al., Applied Physics B 50, 323-329 (1990)

[6] H.M. Lauranto, et al., Spectrochimica Acta Part B 51, 175-180 (1996)

[7] H.M. Lauranto, Appl. Phys. B 66, 231-239 (1998)

[8] H. Tomita, et al., J. Nucl. Sci. Tech. supplyment6, 37-42 (2008)

[9] T. Kessler, et al., Laser Physics 18, 842-849 (2008) 


$$
15^{\text {th }} \text { ISRD }
$$

[10] T. Takatsuka, et al., Hyperfine Interactions 216, 41-46 (2013)

[11] S. Rothe et al., Journal of Physics: Conference Series 312, 052020 (2011)

[12] T. Noto and H. Tomita et al., to be submitted

[13] L. Viererbl et al., Nucl. Instrum. Meth. A 632, 109-111 (2011)

[14] V. Klupák et al., "Nickel foil as transmutation detector for neutron fluence measurements", in ISRD15 proceedings, E153 (2014)

[15] T. Takatsuka, et al., Nucl. Instrum. Meth. B 317, 586-589 (2013) 